\title{
Decentralized simultaneous stabilization and reliable control using periodic feedback *
}

\author{
Konur A. Ünyelioğlu, A. Bülent Özgüler \\ Electrical and Electronics Engineering Department, Bilkent University, 06533, Bilkent, Ankara, Turkey
}

\author{
Pramod P. Khargonekar \\ Department of Electrical Engineering and Computer Science, The University of Michigan, Ann Arbor, MI 48109-2122, USA
}

Received 4 May 1991

Revised 18 September 1991

\begin{abstract}
In this note we consider the simultaneous decentralized stabilization problem for linear, periodically time-varying, discrete time systems. We show that a finite number of periodically time-varying systems can be simultaneously stabilized by a periodically time-varying decentralized compensator if and only if each system admits a decentralized stabilizing compensator. The result is applicable in the design of reliable decentralized stabilizing compensators for interconnected systems.
\end{abstract}

AMS Subject Classification: 93C05, 93B52, 93A14, 93C50, 93D09.

Keywords: Linear systems; decentralized control; time-varying systems; simultaneous stabilization; reliable stabilization.

\section{Introduction}

The following simultaneous decentralized stabilization problem concerning a finite number of periodically time-varying (PTV) plants is considered.

Simultaneous Decentralized Stabilization Problem (SDSP). Given a set of $\nu$-channel, linear, PTV discrete time systems $\Sigma_{1}, \ldots, \Sigma_{k}$ of the same size, determine a decentralized compensator $\bar{\Sigma}=$ bdiag $\left\{\bar{\Sigma}_{1}, \ldots, \bar{\Sigma}_{\nu}\right\}$ such that for all $i=1, \ldots, k$, the pair of plants $\left(\Sigma_{i}, \bar{\Sigma}\right)$ is internally stable.

The main motivation for SDSP is the same as the one given for the centralized simultaneous stabilization problem, [18]. The plants may result by the linearization of a more accurate nonlinear model of the underlying physical system about $k$ different operating points or they may correspond to $k$ possible values attainable by the set of parameters of a parameter dependent system. In the context of large scale interconnected systems, the problem has a special significance [12,17]. Consider an interconnected system which is to be stabilized by a decentralized compensator. A reliable design objective is to maintain closed loop stability under breakdowns in subsystem interconnections. Let us assume that there

\footnotetext{
* Supported in part by National Science Foundation under grants no. NSF-INT-9101276, ECS-9001371, Airforce Office of Scientific Research under contract no. AFOSR-90-0053, Army Research Office under grant no. DAAL03-90-G-0008.
} 
are a finite number of possible breakdowns. Since each breakdown results in a new plant, the decentralized compensator should simultaneously stabilize these plants for reliable operation.

The use of SDSP in reliable decentralized control can be illustrated by the following example. Let the systems $\mathscr{S}_{1}$ and $\mathscr{S}_{2}$ be defined as

$$
\begin{aligned}
& \mathscr{S}_{1}:\left[\begin{array}{l}
x_{1}(t+1) \\
x_{2}(t+1)
\end{array}\right]=\left[\begin{array}{ll}
0 & 1 \\
1 & 1
\end{array}\right]\left[\begin{array}{l}
x_{1}(t) \\
x_{2}(t)
\end{array}\right]+\left[\begin{array}{l}
1 \\
0
\end{array}\right] v_{1}(t), \\
& \mathscr{S}_{2}: \quad x_{3}(t+1)=x_{3}(t)+v_{2}(t),
\end{aligned}
$$

which are interconnected according to the rule $v_{1}(t)=b x_{3}(t)$, where $b$ is a constant. A 2-channel interconnected system $\Sigma_{b}$ out of $\mathscr{S}_{1}, \mathscr{S}_{2}$ is now defined by

$$
\begin{gathered}
\Sigma_{b}:\left[\begin{array}{l}
x_{1}(t+1) \\
x_{2}(t+1) \\
x_{3}(t+1)
\end{array}\right]=\left[\begin{array}{lll}
0 & 1 & b \\
1 & 1 & 0 \\
0 & 0 & 1
\end{array}\right]\left[\begin{array}{l}
x_{1}(t) \\
x_{2}(t) \\
x_{3}(t)
\end{array}\right]+\left[\begin{array}{ll}
1 & 0 \\
0 & 0 \\
0 & 1
\end{array}\right]\left[\begin{array}{l}
u_{1}(t) \\
u_{2}(t)
\end{array}\right], \\
{\left[\begin{array}{l}
y_{1}(t) \\
y_{2}(t)
\end{array}\right]=\left[\begin{array}{lll}
0 & 0 & 1 \\
1 & 0 & 0
\end{array}\right]\left[\begin{array}{l}
x_{1}(t) \\
x_{2}(t) \\
x_{3}(t)
\end{array}\right]}
\end{gathered}
$$

where $\left(u_{1}, y_{1}\right)$ and $\left(u_{2}, y_{2}\right)$ are the input-output pairs associated with Channels 1 and 2, respectively. Assume that the value of $b$ is 1 under normal operation but interconnection between the subsystems $\mathscr{S}_{1}$ and $\mathscr{S}_{2}$ may be subject to a breakdown which results in the value $b=0$. We denote the two different systems that result for these values of $b$ by $\Sigma_{0}$ and $\Sigma_{1}$. To assure reliability, the decentralized controller for $\Sigma_{b}$ should simultaneously stabilize $\Sigma_{0}$ and $\Sigma_{1}$.

The centralized version of the simultaneous stabilization problem has been extensively investigated in the literature for linear, time-invariant (TI) systems using TI controllers $[13,19,18,5]$. It has been shown that $k$ given plants can be simultaneously stabilized if and only if $k-1$ auxiliary plants can be simultaneously stabilized by a stable compensator. For $k=2$, the latter problem has a solution just in case the auxiliary plant satisfies a parity interlacing property. For the general case, however, there are no clear-cut results. On the other hand, any finite collection of TI plants are known to be simultaneously stabilizable by a PTV controller [10,11,7]. The advantage of using PTV controllers in decentralized stabilization is well known (see [2,8] and the references therein). A related problem of simultaneous pole assignment by decentralized feedback has been considered in [7]. Motivated by the advantages of using PTV compensators in simultaneous stabilization and in DSP (Decentralized Stabilization Problem), here we investigate SDSP. The result, Theorem (3.1) below, is somewhat expected but not straightforward to prove. We show that SDSP is solvable if and only if each plant admits a PTV decentralized stabilizing compensator. (The precise conditions under which a given PTV plant admits a decentralized PTV stabilizing controller is given by Theorem 3 of [8].) In other words, 'simultaneous' decentralized stabilization causes no extra problems. Our synthesis procedure is based on the results in [8], [15], and [16] and consists of determining a class of local compensators which, when applied in a prespecified order, simultaneously stabilize the respective channels of the $k$ plants.

The organization of the note is as follows. In Section 2, we review some basic concepts pertinent to the design of decentralized compensators for time-invariant and periodically time-varying systems. Section 3 includes the main result, Theorem 3.1. In Section 4, we give an example to illustrate the synthesis procedure of Theorem 3.1. 


\section{Decentralized controllers for time-invariant and periodically time-varying plants}

Consider a linear, $\nu$-channel, PTV discrete time system

$$
\begin{aligned}
\Sigma: \quad & x(t+1)=A(t) x(t)+\sum_{j=1}^{\nu} B_{j}(t) u_{j}(t), \\
& y_{i}(t)=C_{i}(t) x(t)+\sum_{j=1}^{\nu} D_{i j}(t) u_{j}(t), \quad i=1, \ldots, \nu,
\end{aligned}
$$

where for each nonnegative integer $t, A(t), B_{j}(t), C_{i}(t)$, and $D_{i j}(t), i, j=1, \ldots, \nu$, are real matrices of sizes $n \times n, n \times m_{j}, p_{i} \times n, p_{i} \times m_{j}$, respectively. Let $N$ be the fundamental period of $\Sigma$, i.e., $N$ is the smallest integer for which $A(t+N)=A(t), B_{j}(t+N)=B_{j}(t), C_{i}(t+N)=C_{i}(t)$, and $D_{i j}(t+N)=D_{i j}(t)$, $i, j=1, \ldots, \nu$, for all $t \geq 0$. The system is to be stabilized by a linear, PTV discrete time decentralized compensator of the form

$$
\bar{\Sigma}=\operatorname{diag}\left\{\bar{\Sigma}_{1}, \ldots, \bar{\Sigma}_{\nu}\right\}
$$

where

$$
\begin{aligned}
\bar{\Sigma}_{i}: & \bar{x}_{i}(t+1)=\bar{A}_{i}(t) \bar{x}_{i}(t)+\bar{B}_{i}(t) y_{i}(t), \\
& u_{i}(t)=\bar{C}_{i}(t) \bar{x}_{i}(t)+\bar{D}_{i}(t) y_{i}(t), \quad i=1, \ldots, \nu .
\end{aligned}
$$

For each nonnegative integer $t$, and for $i=1, \ldots, \nu$, the matrices $\bar{A}_{i}(t), \bar{B}_{i}(t), \bar{C}_{i}(t)$, and $\bar{D}_{i}(t)$ are real matrices of sizes $\bar{n}_{i} \times \bar{n}_{i}, \bar{n}_{i} \times p_{i}, m_{i} \times \bar{n}_{i}$, and $m_{i} \times p_{i}$. Let $\bar{\Sigma}$ have the fundamental period $\bar{N}$. Under the assumption that $I-D \bar{D}$ is nonsingular $\forall t \geq 0$, the resulting homogeneous closed-loop system is well-defined and is given by the difference equation

$$
\left[\begin{array}{c}
x(t+1) \\
\bar{x}(t+1)
\end{array}\right]=\left[\begin{array}{cc}
A+B \bar{D}(I-D \bar{D})^{-1} C & B(I-\bar{D} D)^{-1} \bar{C} \\
\bar{B}(I-D \bar{D})^{-1} C & \bar{A}+\bar{B} D(I-\bar{D} D)^{-1} \bar{C}
\end{array}\right]\left[\begin{array}{l}
x(t) \\
\bar{x}(t)
\end{array}\right],
$$

where dependence on $t$ of the matrices $A, \bar{A}$, etc. are not displayed for simplicity and where

$$
\begin{aligned}
& \bar{x}(t):=\left[\begin{array}{c}
\bar{x}_{1}(t) \\
\vdots \\
\bar{x}_{\nu}(t)
\end{array}\right], \\
& \bar{A}:=\operatorname{bdiag}\left\{\bar{A}_{1}, \ldots, \bar{A}_{\nu}\right\}, \quad \bar{B}:=\operatorname{bdiag}\left\{\bar{B}_{1}, \ldots, \bar{B}_{\nu}\right\}, \\
& \bar{C}:=\operatorname{bdiag}\left\{\bar{C}_{1}, \ldots, \bar{C}_{\nu}\right\}, \quad \bar{D}:=\operatorname{bdiag}\left\{\bar{D}_{1}, \ldots, \bar{D}_{\nu}\right\},
\end{aligned}
$$

and

$$
B:=\left[\begin{array}{lll}
B_{1} & \cdots & B_{\nu}
\end{array}\right], \quad C:=\left[\begin{array}{c}
C_{1} \\
\vdots \\
C_{\nu}
\end{array}\right], \quad D:=\left[\begin{array}{ccc}
D_{11} & \cdots & D_{1 \nu} \\
\vdots & & \vdots \\
D_{\nu 1} & \cdots & D_{\nu \nu}
\end{array}\right] .
$$

The decentralized compensator $\bar{\Sigma}$ of (2) internally stabilizes the system $\Sigma$ in (1); equivalently $(\Sigma, \bar{\Sigma})$ is internally stable, if the homogeneous system in (3) is uniformly asymptotically stable. The reader is referred to [6] and [21] for further details of definition of stability for time-varying systems. 
If the system in (1) is time-invariant, that is $N=1$, then $\Sigma$ admits a time-invariant decentralized stabilizing compensator if and only if it has no unstable decentralized fixed modes, equivalently, if and only if

$$
\operatorname{rank}\left[\begin{array}{ccccc}
z I-A & B_{j_{1}} & B_{j_{2}} & \cdots & B_{j_{\nu-\mu}} \\
C_{i_{1}} & D_{i_{1} j_{1}} & D_{i_{1} j_{2}} & \cdots & D_{i_{1} j_{\nu-\mu}} \\
C_{i_{2}} & D_{i_{2} j_{1}} & D_{i_{2} j_{2}} & \cdots & D_{i_{2} j_{\nu-\mu}} \\
\vdots & \vdots & \vdots & & \vdots \\
C_{i_{\mu}} & D_{i_{\mu} j_{1}} & D_{i_{\mu} j_{2}} & \cdots & D_{i_{\mu} j_{\nu-\mu}}
\end{array}\right] \geq n,
$$

for all $\mu=1, \ldots, \nu$ and $i_{k}, j_{l} \in\{1, \ldots, \nu\}$ such that $\left\{i_{1}, \ldots, i_{\mu}, j_{1}, \ldots, j_{\nu-\mu}\right\}=\{1, \ldots, \nu\}$ (see $[3,1,4]$, and also [20]). The subsystems of (1) corresponding to the system matrix in (4) are called the complementary subsystems of (1) [3]. In [15], a new design procedure of decentralized stabilizing compensators is proposed for systems which are free of unstable decentralized fixed modes. It is shown that almost any compensator which internally stabilizes the $\nu$-th channel of the system (i.e., the $\nu$-th diagonal transfer matrix) the resulting $\nu-1$-channel closed loop system is also free of unstable decentralized fixed modes. And hence, at each step, the local compensator to be applied to the respective channel can be chosen almost freely in the set of internally stabilizing compensators of that channel. To be more precise, let $Z=\left[Z_{i j}\right]$ be the transfer matrix of the TI system (1), where $Z_{i j}=C_{i}(z I-A)^{-1} B_{j}+D_{i j}, i, j=1, \ldots, \nu$. Let $\mathcal{Z}_{c}\left(Z_{\nu \nu}\right)$ be the set of all proper TI internally stabilizing compensators of $Z_{\nu \nu}$. Using the graph topology [18] induced by the fractional representation of transfer matrices over the ring of stable proper transfer functions $\mathbf{S}$, one can associate a suitable topology for the set $\mathcal{Z}\left(Z_{\nu \nu}\right)[15,16]$. The quantifier 'almost' below is with respect to this topology.

Theorem 2.1. Let the time-invariant plant in (1) be free of unstable decentralized fixed modes. Then, for almost all $\bar{Z}_{\nu} \in \mathcal{Z}_{\mathrm{c}}\left(Z_{\nu \nu}\right)$, applied around channel $\nu$, the resulting $\nu-1$ channel closed loop system $\hat{\Sigma}$ is also free of unstable decentralized fixed modes.

Remark 2.1. The actual statement in Theorem 1 of [15] is given under the assumption that the diagonal subplants of the system is strictly proper. However, this assumption can be removed easily as in [16].

Remark 2.2. Let $\bar{Z}_{\nu}$ be a local compensator applied around channel $\nu$ and let a stabilizable and detectable realization of $\bar{Z}_{\nu}$ be $\left(F_{\nu}, G_{\nu}, H_{\nu}, J_{\nu}\right)$. If the resulting $\nu-1$ channel system $\hat{\Sigma}$ is not free of unstable decentralized fixed modes, the theorem states the existence of another compensator $\bar{Z}_{v \Delta}$, which is arbitrarily close to $\bar{Z}_{\nu}$ in the graph topology, and for which the resulting closed loop system is free of unstable decentralized fixed modes. A crucial point is the following: one can choose $\bar{Z}_{\nu \Delta}$ in such a way that any realization of $\left(G_{\Delta}, G_{\Delta}, H_{\Delta}, J_{\Delta}\right)$ satisfies $J_{\Delta}=J_{\nu}$. In other words, the transfer matrices of the perturbed and nominal compensators take the same value at $z=\infty$. This property will be used in the construction part of Theorem 3.1.

Let us now consider decentralized stabilization of PTV systems by PTV controllers. A convenient tool here is the procedure of lifting [8]. Consider a $p \times m$ PTV system $\Sigma$ given by

$$
\begin{aligned}
\Sigma: \quad & x(t+1)=A(t) x(t)+B(t) u(t), \\
& y(t)=C(t) x(t)+D(t) u(t), \quad t \geq 0,
\end{aligned}
$$

with fundamental period $N$. Any multiple $M$ of $N$ with a positive integer is also a period and we define the $M$-lifting of $\Sigma$ to be the time invariant (TI) $p M \times m M$ system $\Sigma^{M}$ given by the equations

$$
\begin{aligned}
\Sigma^{M}: \quad & \hat{x}(t+1)=F \hat{x}(t)+G \hat{u}(t), \\
& \hat{y}(t)=H \hat{x}(t)+J \hat{u}(t), \quad t \geq 0,
\end{aligned}
$$


where the constant matrices $F, G, H, J$ are given in terms of the state transition matrix

$$
\Phi(t, l):= \begin{cases}A(t-1) \ldots A(l) & \text { if } t>l, \\ l & \text { if } t=l, \\ \text { undefined } & \text { if } t<l,\end{cases}
$$

as follows:

$$
\begin{aligned}
& F:=\Phi(M, 0), \\
& G:=[\Phi(M, 1) B(0) \quad \Phi(M, 2) B(1) \quad \cdots \quad \Phi(M, M-1) B(M-2) \quad B(M-1)], \\
& H:=\left[\begin{array}{c}
C(0) \\
C(1) \Phi(1,0) \\
\vdots \\
C(M-2) \Phi(M-2,0) \\
C(M-1) \Phi(M-1,0)
\end{array}\right],
\end{aligned}
$$

and the matrix $J=\left[J_{i j}\right]$ is such that

$$
J_{i j}= \begin{cases}0 & \text { if } i<j \\ D(i-1) & \text { if } i=j \\ C(i-1) \Phi(i-1, j) B(j-1) & \text { if } i>j\end{cases}
$$

for $i, j=1, \ldots, M$.

The following lemma transforms the problem of internal stabilization of a PTV system using a PTV controller to that of a TI system using a TI controller.

Lemma 2.1. Let $\Sigma$ be a PTV system with period $M$ and let $\Sigma^{M}$ be its $M$-lifting. There exists a PTV controller internally stabilizing $\Sigma$ if and only if there exists a TI controller internally stabilizing $\Sigma^{M}$.

Proof. For the details the reader is referred to [10] (see also [8]). We just summarize the main steps in the proof. If there exists a PTV controller internally stabilizing $\Sigma$, then its $M$-lifting also internally stabilizes $\Sigma^{M}$, the $M$-lifting of $\Sigma$. This is a straightforward consequence of definitions. Conversely, if there exists a TI internally stabilizing compensator for $\Sigma^{M}$, then there also exists a strictly proper TI internally stabilizing compensator for $\Sigma^{M}$, which can then be transformed to a PTV internally stabilizing compensator for $\Sigma$, by a reverse procedure of lifting, which we call sinking. The reason for the insistence on the strict properness of the compensator is that, only those compensators which have a suitable lower triangular form at $z=\infty$ can be sunk to yield PTV compensators.

Let us now consider the $\nu$-channel PTV system $\Sigma$ of (1). We are now interested in the existence of a decentralized PTV compensator of the form (2) which stabilizes $\Sigma$. The following is Theorem 3 of [8].

Theorem 2.2. There exists a PTV decentralized controller internally stabilizing the PTV system $\Sigma$ of (1) if and only if $\Sigma$ is stabilizable and detectable and for each complementary subsystem of $\Sigma$,

$$
\begin{aligned}
f\left(\Sigma\left\{i_{1}, \ldots, i_{\mu}, j_{1}, \ldots, j_{\nu-\mu}\right\}\right)=0 \Rightarrow & \Sigma^{N}\left\{i_{1}, \ldots, i_{\mu}, j_{1}, \ldots, j_{\nu-\mu}\right\} \text { has no unstable } \\
& \text { input-output decoupling zeros, }
\end{aligned}
$$

where $f\left(\Sigma\left\{i_{1}, \ldots, i_{\mu}, j_{1}, \ldots, j_{\nu-\mu}\right\}\right)$ is the input-output map of the complementary subsystem $\Sigma\left\{i_{1}, \ldots, i_{\mu}, j_{1}, \ldots, j_{\nu-\mu}\right\}$ of $\Sigma$ and $\Sigma^{N}\left\{i_{1}, \ldots, i_{\mu}, j_{1}, \ldots, j_{\nu-\mu}\right\}$ is its $N$-lifting. 
Remark 2.3. If (7) is satisfied for all complementary subsystems of $\Sigma$, then there exists a multiple $\bar{M}$ of $N$ (which can be determined from (1) in a straightforward manner) such that the $M$-lifted time-invariant systems in (8) are free of unstable decentralized fixed modes, provided $M \geq \bar{M}$. (See Theorem 1 of [8].)

\section{Main results}

For the main result Theorem 3.1 of this note we first prove the following preliminary result concerned with centralized (full-feedback) simultaneous stabilization.

Lemma 3.1. Any finite collection of PTV systems of the same size can be simultaneously stabilized by a centralized PTV controller.

Proof. This is a discrete-time counterpart of the result of [11] and of Theorem 4.7 of [9]. We give a proof which closely follows the proof of Theorem 4.2 in [10]. Let $\Sigma_{1}, \ldots, \Sigma_{k}$ be a given set of PTV plants. Let $M$ be the least common multiple of their fundamental periods. Using the procedure of Theorem 4.2 in [10], one can construct a PTV controller $\bar{\Sigma}$ which simultaneously stabilizes the $M$-liftings $\Sigma_{1}^{M}, \ldots, \Sigma_{k}^{M}$, of the given plants. In particular one can choose $\bar{\Sigma}=(\bar{A}, \bar{B}, \bar{C}, \bar{D})$ with $\bar{D}=0$. Let $M_{\mathrm{c}}$ be the fundamental period of $\bar{\Sigma}$ and define $\bar{M}$ to be the least common multiple of $M_{\mathrm{c}}$ and $M$. Observe that the $\bar{M}$-lifting $\bar{\Sigma}^{\bar{M}}$ of the compensator has a transfer matrix which takes a lower triangular form at $z=\infty$. The TI compensator $\bar{\Sigma}^{\bar{M}}$ also internally stabilizes the TI plants $\Sigma_{1}^{\bar{M}}, \ldots, \Sigma_{k}^{\bar{M}}$. From Lemma $2.1, \bar{\Sigma}^{\bar{M}}$ can be sunk to a PTV system with period $\bar{M}$, which internally stabilizes the desired set of plants. This completes the proof.

We now give our main result.

Theorem 3.1. SDSP is solvable if and only if each of $\Sigma_{i}, i=1, \ldots, k$, can be internally stabilized by $a$ PTV decentralized compensator.

Proof. The necessity is obvious. To show sufficiency, we first introduce a notation. Let $\left(F, G_{j}, H_{i}, J_{i j}\right)$ be the $M$-lifting of the subsystem $\left(A(t), B_{j}(t), C_{i}(t), D_{i j}(t)\right)$ of (1) for some multiple $M$ of $N$ and for $i$, $j=1, \ldots, \nu$. The $\nu$-channel time invariant system obeying

$$
\begin{array}{ll}
\Sigma^{M, \pi}: \quad & \hat{x}(t+1)=F \hat{x}(t)+\sum_{j=1}^{\nu} G_{j} \hat{u}_{j}(t), \\
& \hat{y}_{i}(t)=H_{i} \hat{x}(t)+\sum_{j=1}^{\nu} J_{i j} \hat{u}_{j}(t), \quad i=1, \ldots, \nu,
\end{array}
$$

is the $M$-lifting of the original system (1) modulo some permutations of the input and output channels. The superscript $\pi$ is thus included to emphasize the fact that $\Sigma^{M, \pi}$ is an $M$-lifting of the system (1) followed by a permutation at the input and output channels. The significance of $\Sigma^{M, \pi}$ is the following: The plant (1) is stabilizable by a decentralized PTV controller if and only if $\Sigma^{M, \pi}$ is stabilizable by a TI decentralized controller. The permutation at the input and output channels of $\Sigma^{M}$ is thus necessary to preserve the decentralized structure of the controller (see [8]).

Let a PTV compensator $\Sigma_{\mathrm{c}}$ of period $N_{\mathrm{c}}$ simultaneously stabilize channel $\nu$ of all $\Sigma_{i}, i=1, \ldots, k$. Such a compensator can be constructed using Lemma 3.1. By the hypothesis and Theorem 2.2, for $i=1, \ldots, k$, one can determine $M_{i}$ such that the TI system $\Sigma_{i}^{M_{i}, \pi}$ is free of unstable decentralized fixed modes, where $\Sigma_{i}^{M_{i}, \pi}$ is obtained from the $M_{i}$-lifting $\Sigma_{i}^{M_{i}}$ of the system $\Sigma_{i}$ as above (see also Remark 2.3). Let $M$ be the least common multiple of the integers $\left\{N_{c}, M_{1}, \ldots, M_{k}\right\}$. Then, from the definition of lifting, the $M$-lifting $\Sigma_{\mathrm{c}}^{M}$ of $\Sigma_{\mathrm{c}}$, also simultaneously stabilizes channel $\nu$ of all $\Sigma_{i}^{M, \pi}, i=1, \ldots, k$. Let $Z_{\mathrm{c}}^{M}$ 
be the transfer matrix of $\Sigma_{\mathrm{c}}^{M}$. From Theorem 2.1 and the following discussion, by suitably perturbing $Z_{\mathrm{c}}^{M}$ to $Z_{\Delta c}^{M}$ if necessary, the resulting $\nu-1$ channel TI closed loop systems $\hat{\Sigma}_{i}^{M, \pi}$ for $i=1, \ldots, k$ are free of unstable decentralized fixed modes. The perturbation on $Z_{\mathrm{c}}^{M}$ can be chosen such that the transfer matrices $Z_{\Delta \mathrm{c}}^{M}$ and $Z_{\mathrm{c}}^{M}$ take the same value at $z=\infty$ (see Remark 2.2). So, from Lemma 2.1, $Z_{\Delta \mathrm{c}}^{M}$ has a PTV realization. Also for $i=1, \ldots, k$, let $\hat{\Sigma}_{i}$ be the $M$-sinking of $\hat{\Sigma}_{i}^{M}$, where $\hat{\Sigma}_{i}^{M}$ is obtained from $\hat{\Sigma}_{i}^{M, \pi}$, by re-permuting the inputs and outputs appropriately, so as to obtain the same input-output structure as the $M$-lifting $\Sigma_{i}^{M}$ of $\Sigma_{i}$. The discussion at the beginning of proof and the fact that $\hat{\Sigma}_{i}^{M, \pi}$ for $i=1, \ldots, k$ are free of unstable decentralized fixed modes imply that each $\hat{\Sigma}_{i}$ for $i=1, \ldots, k$ admits a PTV decentralized stabilizing compensator. Also observe that each $\hat{\Sigma}_{i}$ has $\nu-1$ channels. Now repeating this procedure $\nu-1$ times, one finally obtains a PTV decentralized simultaneously stabilizing compensator of the form (2), for $\Sigma_{i}, i=1, \ldots, k$.

If each $\Sigma_{1}, \ldots, \Sigma_{k}$ is stabilizable and detectable and strongly connected [3], then by Theorem 2.2, they all admit stabilizing PTV decentralized controllers. Thus we obtain the following corollary of Theorem 3.1.

Corollary 3.1. SDSP has a solution for any finite collection of stabilizable and detectable, and strongly connected PTV systems.

Remark 3.1. An alternative procedure for constructing a simultaneously stabilizing decentralized controller can be given if the systems $\Sigma_{i}, i=1, \ldots, k$, satisfy an extra condition. We first note the following:

For the system in (1) a PTV dead-beat decentralized controller exists, in other words for some decentralized controller of the type (2), the closed loop state matrix $\hat{A}(t)$ of the homogeneous system in (3) satisfies $[\hat{A}(M-1) \hat{A}(M-2) \cdots \hat{A}(0)]^{q}=0$, for some integer $q$, if and only if $\Sigma$ is stabilizable and detectable and for each complementary subsystem of $\Sigma$,

$$
\begin{aligned}
f\left(\Sigma\left\{i_{1}, \ldots, i_{\mu}, j_{1}, \ldots, j_{\nu-\mu}\right\}\right)=0 \Rightarrow & \Sigma^{N}\left\{i_{1}, \ldots, i_{\nu}, j_{1}, \ldots, j_{\nu-\mu}\right\} \text { has no unstable } \\
& \text { input-output decoupling zeros except } 0,
\end{aligned}
$$

where we use the same notation as in Theorem 2.2 .

If each of the systems $\Sigma_{i}, i=1, \ldots, k$, admit a PTV dead-beat decentralized controller, then these controllers can be run in a cycle to achieve a simultaneously stabilizing decentralized controller. This procedure has been used in $[10,11]$ in the simultaneous stabilization problem with centralized compensators.

\section{Example}

Consider the example in the first section. $\Sigma_{0}$ and $\Sigma_{1}$ have an unstable decentralized fixed mode at $z=1$ (see [14]). However the systems are both stabilizable and detectable and moreover the input-output maps $\Sigma_{0}\{1,2\}, \Sigma_{0}\{2,1\}, \Sigma_{1}\{1,2\}, \Sigma_{1}\{2,1\}$ are all nonzero so that both systems are strongly connected. By Corollary 3.1, $\Sigma_{0}$ and $\Sigma_{1}$ can be simultaneously stabilized by a PTV decentralized compensator. Define a 2-periodic compensator $\Sigma_{\mathrm{c}}=\left(\bar{A}_{1}, \bar{B}_{1}, \bar{C}_{1}, \bar{D}_{1}\right)$ as follows: $\bar{A}_{1}(t)=0, \forall t \geq 0 ; \quad \bar{B}_{1}(2 t)=2$, $\bar{B}_{1}(2 t+1)=0, \forall t \geq 0 ; \bar{C}_{1}(t)=1, \forall t \geq 0 ; \bar{D}_{1}(2 t)=3, \bar{D}_{1}(2 t+1)=0, \forall t \geq 0$. $\Sigma_{\text {c }}$ simultaneously stabilizes channel 2 of $\Sigma_{1}$ and $\Sigma_{2}$. Also the resulting single channel PTV closed loop systems $\bar{\Sigma}_{0}$ and $\bar{\Sigma}_{1}$ are stabilizable and detectable from channel 1. We now design a PTV controller that stabilizes $\bar{\Sigma}_{0}$ and $\bar{\Sigma}_{1}$ by 
using the procedure in Lemma 3.1. The 2-liftings $\Sigma_{0}^{2 . \pi}$ and $\Sigma_{1}^{2 . \pi}$ of $\Sigma_{0}$ and $\Sigma_{1}$ followed by the permutations at the inputs and outputs are given by

$$
\begin{array}{ll}
A=\left[\begin{array}{lll}
1 & 1 & b \\
1 & 2 & b \\
0 & 0 & 1
\end{array}\right], & B_{1}=\left[\begin{array}{ll}
0 & 1 \\
1 & 0 \\
0 & 0
\end{array}\right], \quad B_{2}=\left[\begin{array}{ll}
b & 0 \\
0 & 0 \\
1 & 1
\end{array}\right] \\
C_{1}=\left[\begin{array}{lll}
0 & 0 & 1 \\
0 & 0 & 1
\end{array}\right], & C_{2}=\left[\begin{array}{lll}
1 & 0 & 0 \\
0 & 1 & b
\end{array}\right], \quad D_{11}=D_{22}=0_{2 \times 2}, \quad D_{12}=D_{21}=\left[\begin{array}{ll}
0 & 0 \\
1 & 0
\end{array}\right],
\end{array}
$$

for $b=0$ and $b=1$, respectively. The 2-lifting $\Sigma_{c}^{2}$ of $\Sigma_{c}$ is computed as

$$
\left(0,\left[\begin{array}{ll}
0 & 0
\end{array}\right],\left[\begin{array}{ll}
0 & 0
\end{array}\right]^{\prime},\left[\begin{array}{ll}
3 & 0 \\
2 & 0
\end{array}\right]\right) \text {. }
$$

The single channel closed loop transfer matrices corresponding to 2-lifted TI systems $\bar{\Sigma}_{0}^{2, \pi}$ and $\bar{\Sigma}_{1}^{2, \pi}$ are given by

$$
\left[\begin{array}{cc}
\frac{-5}{z^{3}+z^{2}(3 b-4)+z(4-14 b)+21 b-1} & \frac{-5(z-2)}{z^{3}+z^{2}(3 b-4)+z(4-14 b)+21 b-1} \\
\frac{-(3 z+2)}{z^{3}+z^{2}(3 b-4)+z(4-14 b)+21 b-1} & \frac{-\left(3 z^{2}-4 z-4\right)}{z^{3}+z^{2}(3 b-4)+z(4-14 b)+21 b-1}
\end{array}\right]
$$

for $b=0$ and $b=1$ respectively. The 10 -periodic compensator $\hat{\Sigma}=(\hat{A}, \hat{B}, \hat{C}, 0)$ simultaneously stabilizes $\bar{\Sigma}_{1)}^{2 . \pi}$ and $\bar{\Sigma}_{1}^{2, \pi}$, where

$$
\begin{aligned}
& \hat{A}(10 i+t)= \begin{cases}{\left[\begin{array}{ll}
0 & 0 \\
0 & -1
\end{array}\right],} & \\
{\left[\begin{array}{cc}
-3.3352 & 0 \\
0 & -0.6648
\end{array}\right],} & t \in\{5,6,7,8,9\}, \quad \forall i,\end{cases} \\
& \hat{B}(t)=I_{2 \times 2}, \quad \forall t, \\
& \hat{C}(10 i+t)= \begin{cases}{\left[\begin{array}{ll}
-5.3333 & 0 \\
-0.2667 & -0.3333
\end{array}\right],} & t \in\{0,1,2,3,4\}, \\
{\left[\begin{array}{ll}
-3.4687 & 0 \\
5.1429 & -3.2609
\end{array}\right],} & t \in\{5,6,7,8,9\}, \quad \forall i .\end{cases}
\end{aligned}
$$

The 10-lifting of $\hat{\Sigma}$ is the 20-lifting of a 20-periodic compensator $\tilde{\Sigma}$, which can be obtained via the procedure of sinking described in Lemma 5 in [8]. Thus the decentralized compensator of period 20 consisting of $\tilde{\Sigma}_{\mathrm{c}}$ and $\Sigma_{\mathrm{c}}$ around channels 1 and 2 solves SDSP for $\Sigma_{0}$ and $\Sigma_{1}$. As this example shows, the synthesis procedure proposed by Theorem 2.2 may lead to rather large periods as no attempt has been made to optimize the procedure with respect to the period or to the dimension of the resulting controller.

\section{References}

[1] B.D.O. Anderson and D.J. Clements, Algebraic characterization of fixed modes in decentralized control, Automatica 17 (1981) $703-712$.

[2] B.D.O. Anderson and J.B. Moore, Time-varying feedback laws for the decentralized control, IEEE Trans. Automat. Control 26 (1981) 1133-1139.

[3] J.P. Corfmat and A.S. Morse, Decentralized control of linear multivariable systems, Automatica 8 (1976) $479-485$.

[4] E.J. Davison and T.N. Chang, Decentralized stabilization and pole assignment for general proper systems, IEEE Trans. Automat. Control 35 (1990) 652-664. 
[5] B.K. Ghosh, An approach to simultaneous system design. Part II: Nonswitching gain and dynamic feedback compensation by algebraic geometric methods, SIAM J. Control Optim. 26 (1988) 919-963.

[6] W. Hahn, Stability of Motion (Springer-Verlag, Berlin-New York, 1967).

[7] Y.C. Juan and P.T. Kabamba, Simultaneous pole assignment in linear periodic systems by constrained structure feedback, IEEE Trans. Automat. Control 34 (1989) 168-173.

[8] P.P. Khargonekar and A.B. Özgüler, Decentralized control and periodic feedback, Submitted for publication (1991).

[9] P.P. Khargonekar, A.M. Pascoal, and R. Ravi, Strong, simultaneous and reliable stabilization of finite-dimensional time-varying plants, IEEE Trans. Automat. Control 33 (1988) 1158-1161.

[10] P.P. Khargonekar, K. Poolla, and A. Tannenbaum, Robust control of linear time-invariant plants using periodic compensation, IEEE Trans. Automat. Control 30 (1985) 1088-1096.

[11] A.W. Olbrot, Robust stabilization of uncertain systems by periodic feedback, Internat. J. Control 45 (1987) 747-758.

[12] A.B. Özgüler and M. Hıraoğlu, Implications of a characterization result on strong and reliable decentralized control, in: R.F. Curtain, Ed., Modelling, Robustness and Sensitivity Reduction in Control Systems, NATO ASI Series Vol. F34 (Springer-Verlag, Berlin, 1987) 425-450.

[13] R. Saeks and J. Murray, Fractional representation, algebraic geometry and the simultaneous stabilization problem, IEEE Trans. Automat. Control 26 (1981) 203-218.

[14] M.E. Sezer and D.D. Šiljak, Structurally fixed modes, Systems Control Lett. 1 (1981) 60-64.

[15] K.A. Ünyelioğlu and A.B. Özgüler, Decentralized stabilization of multivariable systems using a stable proper fractional approach, in: E. Arıkan, Ed., Proceedings of the 1990 Bilkent International Conference on Communication, Control and Signal Processing, Vol. 1 (Elsevier, Ankara, 1990) 843-849.

[16] K.A. Ünyelioğlu and A.B. Özgüler, Decentralized stabilization: characterization of all solutions and genericity aspects, Internat. J. Control (1991), to appear.

[17] K.A. Ünyelioğlu and A.B. Özgüler, Reliable decentralized stabilization of feedforward and feedback interconnected systems, to appear.

[18] M. Vidyasagar, Control System Synthesis (The MIT Press, Cambridge, 1985).

[19] M. Vidyasagar and N. Viswanadham, Algebraic design techniques for reliable stabilization, IEEE Trans. Automat. Control 27 (1982) 856-859.

[20] S.H. Wang and E.J. Davison, On the stabilization of decentralized control systems, IEEE Trans. Automat. Control 18 (1973) 473-478.

[21] J.L. Willems, Stability Theory of Dynamical Systems (John Wiley, New York, 1970). 